\title{
A Note on - Common Fixed Points of Faintly Compatible Mappings
}

\author{
Saurabh Manro ${ }^{1}$ \\ School of Mathematics and Computer Applications, Thapar University, Patiala, Punjab, India \\ Corresponding Address: B.No. 33, H.No. 223, Peer Khana Road, Near Tiwari Di Kothi, Khanna, District- \\ Ludhiana, State- Punjab-141401
}

\begin{abstract}
:
The aim of this note is to point out errors in Theorems 3.1, 3.4, 3.6 and hence in Corollary 3.7 of the paper [2] proved in metric spaces. We also rectify errors in these results by replacing a pair of reciprocal continuous self mappings by conditionally reciprocally continuous mappings.
\end{abstract}

Keywords: Conditional reciprocal continuity, compatible maps, faintly compatible maps, reciprocal continuous maps, common fixed points.

\section{INTRODUCTION AND PRELIMINARIES}

In order to avoid repetition, we assume the terminology and the notations utilized in [1,2].

Definition 1.1. A pair of self-maps $(A, S)$ on a metric space $(X, d)$ is said to be compatible, if $\lim _{\mathrm{n}} d\left(A S x_{n}, S A x_{n}\right)=0$, whenever $\left\{x_{n}\right\}$ is a sequence in $X$ such that $\operatorname{limn} A x_{n}=\lim _{\mathrm{n}} S x_{\mathrm{n}}=x$, for some $x$ in $X$.

Definition 1.2. A pair of self-maps $(A, S)$ on a metric space $(X, d)$ is said to be non-compatible, if $(A, S)$ is not compatible, i.e., if there exists a sequence $\left\{x_{n}\right\}$ in $X$ such that $\lim _{\mathrm{n}} A x_{n}=\lim _{\mathrm{n}} S x_{\mathrm{n}}=x$, for some $x$ in $X$ and $\lim _{\mathrm{n}}$ $d\left(A S x_{n}, S A x_{n}\right) \neq 0$, or non-existent.

Definition 1.3. A pair of self-maps $(A, S)$ on a metric space $(X, d)$ is said to be weakly compatible, if the pair commute on the set of their coincidence points, i.e., for $x$ in $X$; $A x=S x$ implies $A S x=S A x$.

Definition 1.4. A pair of self-maps $(A, S)$ on a metric space $(X, d)$ is said to be conditionally compatible, iff whenever the set of sequences $\left\{x_{n}\right\}$ satisfying $\lim _{\mathrm{n}} A x_{n}=\lim _{\mathrm{n}} S x_{\mathrm{n}}$, is non-empty, there exists a sequence $\left\{z_{n}\right\}$ in $X$ such that $\lim _{\mathrm{n}} A z_{n}=\lim _{\mathrm{n}} S z_{\mathrm{n}}$ and $\lim _{\mathrm{n}} d\left(A S z_{n}, S A z_{n}\right)=0$.

Definition 1.5. A pair of self-maps $(A, S)$ on a metric space $(X, d)$ is said to be faintly compatible, iff $(A, S)$ is conditionally compatible and $A$ and $S$ commute on a non-empty subset of the set of coincidence points, whenever the set of coincidence points is nonempty.

Definition 1.6. A pair of self-maps $(A, S)$ on a metric space $(X, d)$ is said to be reciprocally continuous, if $\lim _{\mathrm{n}} A S x_{n}$ $=A x, \lim _{\mathrm{n}} S A x_{n}=S x$, whenever $\left\{x_{n}\right\}$ is a sequence in in $X$ such that $\lim _{\mathrm{n}} A x_{n}=\lim _{\mathrm{n}} S x_{\mathrm{n}}=x$, for some $x$ in $X$.

Definition 1.7. A pair of self-maps $(A, S)$ on a metric space $(X, d)$ is said to be conditionally reciprocal continuous iff whenever the set of sequences $\left\{x_{n}\right\}$ satisfying $\lim _{\mathrm{n}} A x_{n}=\lim _{\mathrm{n}} S x_{\mathrm{n}}$ is nonempty, there exists a sequence $\left\{y_{n}\right\}$ satisfying $\lim _{\mathrm{n}} A y_{\mathrm{n}}=\lim _{\mathrm{n}} S y_{\mathrm{n}}=x$ (say) for some $x$ in $X$ such that $\lim _{\mathrm{n}} A S y_{n}=A x$ and $\operatorname{limn} S A y_{n}=S x$.

\section{Main Results}

Utilizing the concept of reciprocal continuity property of mappings, M. L. Saha et al. [2] proved Theorems 3.1, 3.4, 3.6 and hence in Corollary 3.7 in metric spaces.

Unfortunately, Theorems 3.1, 3.4, 3.6 and hence in Corollary 3.7 are not true in its present form. On critical examination of the results given in paper [2], we notice one crucial error. We need to carry out the following correction:

Proof of Theorems 3.1, 3.4, 3.6 and hence in Corollary 3.7 given in paper [2] are wrong as proofs of these Theorems and Examples 3.2, 3.5 suggest that a pair of self $(A, S)$ is not reciprocal continuous however it is conditionally reciprocally continuous.

\footnotetext{
${ }^{1}$ Corresponding Author: sauravmanro@hotmail.com
} 
It is not easy to overcome this problem. In order to overcome this problem, one should replace the assumption- ( $A$, $S$ ) is reciprocal continuous in Theorems $3.1,3.4,3.6$ and hence in Corollary 3.7 by the stronger one: $(A, S)$ is conditionally reciprocally continuous mappings [1].

\section{CONCLUSION}

In this note, we point out errors in Theorems 3.1, 3.4, 3.6 and hence in Corollary 3.7 of the paper [2] proved in metric spaces. We also rectify errors in these results by replacing a pair of reciprocal continuous self mappings by conditionally reciprocally continuous mappings.

\section{REFERENCES}

[1] R.K. Bisht and R.P. Pant, Common fixed point theorems under a new continuity condition, Ann. University Ferrara, 58 (2012), 127-141.

[2] M.L. Saha and S. Manro, Common fixed points of faintly compatible mappings, American Research Journal of Mathematics, 1(2)(2015), 38-43. 